\title{
HOXD-AS1 promotes the epithelial to mesenchymal transition of ovarian cancer cells by regulating miR-186-5p and PIK3R3
}

Shanshan Dong ${ }^{1,2 \dagger}$, Ranran Wang ${ }^{1,2 \dagger}$, Hui Wang ${ }^{3}$, Qi Ding ${ }^{1,2}$, Xiao Zhou ${ }^{1,2}$, Jing Wang ${ }^{4}$, Keqiang Zhang ${ }^{4}$, Ying Long ${ }^{1}$, Shan Lü ${ }^{5}$, Ting Hong ${ }^{4}$, Huayi Ren ${ }^{1}$, Kee Wong ${ }^{2}$, Xiaowu Sheng ${ }^{1,2}$, Yu Wang ${ }^{6 *}$ and Yong Zeng ${ }^{1,2^{*}}$ (D)

\begin{abstract}
Background: Epithelial ovarian cancer (EOC) is one of the most malignant gynecological tumors worldwide. Deregulation of long non-coding RNAs (IncRNAs) has been implicated in various oncogenic processes in multiple cancers. In this study, we aim to identify and characterize clinically relevant IncRNA deregulation in EOC.

Methods: LncRNAs, mRNAs and miRNAs were profiled using expression microarrays and validated using reverse transcription quantitative PCR in EOC cells and tissues. siRNAs targeting either HOXD-AS1 or PIK3R3 together with miR-186-5p inhibitors were used to modulate endogenous target expression in EOC cell lines in vitro. In vitro wound healing assay, trans-well assay, Western-blot assay,and Dual-luciferase reporter assay were used to explore the biological roles and molecular function underlying HOXD-AS1 in the EOC cells. Progression-free survival (PFS) and overall survival (OS) were statistically analyzed by Kaplan-Meier method test.
\end{abstract}

Results: HOXD-AS1 was found to be significantly over-expressed in EOC tumors. High HOXD-AS1 expression significantly correlated with poorer PFS and OS of EOC patients. Multivariate Cox proportional hazards modeling indicated that HOXD-AS1 was an independent risk predictor of EOC patients ( $H R=1.92, p=0.004)$. SiRNA inhibition of HOXD-AS1 reduced cell migration, invasion, and epithelial-mesenchymal transition (EMT) in EOC cells in vitro by preventing HOXD-AS1 directly binding to miR-186-5p, and resulting in down-regulating of PIK3R3. The novel HOXD-AS1/miR-186-5p/PIK3R3 pathway was clinically relevant as we observed a significantly inverse correlation between HOXD-AS1/miR-186-5p and between miR-186-5p/PIK3R3 in an independent cohort of 200 EOC tissues.

Conclusions: HOXD-AS1/miR-186-5p/PIK3R3 is a novel pathway to promote cell migration, invasion, and EMT in EOC.

Keywords: HOXD-AS1, EMT, miR-186-5p, PIK3R3, IncRNA, ceRNA, EOC

\section{Background}

EOC is the third most common and second most lethal gynecological malignancy worldwide [1]. The standard treatment is maximal cytoreductive surgery followed by platinum-based chemotherapy regimen [2]. However, the occurrence of metastasis and acquired drug resistance often lead to poor prognosis in patients with advanced

\footnotetext{
*Correspondence: valzeng@sina.cn; wang.yu@nccs.com.sg

†'Shanshan Dong and Ranran Wang contributed equally to this work.

${ }^{1}$ Translational Medicine Center, The Affiliated Cancer Hospital of Xiangya

School of Medicine, Central South University/Hunan Cancer Hospital,

Changsha, China

${ }^{6}$ Division of Cellular and Molecular Research, National Cancer Centre

Singapore, Singapore, Singapore

Full list of author information is available at the end of the article
}

ovarian cancer [3, 4]. Therefore, there is an urgent clinical need to understand the molecular mechanisms leading to the occurrence of tumor metastasis and drug resistance in EOC. In addition, novel biomarkers which can accurately predict tumor metastasis and drug resistance are needed to provide valuable insight into the clinical management of EOC.

EMT is a biological process in which polarized epithelial cells adopt mesenchymal cell phenotype which includes enhanced migratory capacity, invasiveness, elevated resistance to apoptosis, and greatly increased production of extracellular matrix components $[5,6]$. It has been well demonstrated that the activation of EMT program endows cancer cells with the multiple features that are required to 
execute most steps of the invasion-metastasis cascade $[7,8]$. In addition, activation of EMT pathway induces chemoresistance in multiple cancers such as pancreatic cancer [9], lung cancer [10, 11], gastric cancer [12], liver cancer [13] and EOC [14].

LncRNA and miRNAs both belong to non-protein encoding RNAs which are implicated to play important roles in a variety of cellular processes such as cell proliferation, apoptosis, and migration $[15,16]$. The aberrant expression of lncRNAs in tumors can induce EMT and increase the invasion and migration of tumor cells. For example, XIST, which is a lncRNA over-expressed in colorectal cancer tissues and cells, can promote invasion, proliferation, and EMT progress through XIST/miR200b-3p/ZEB1 pathway [17]. Another example is PVT1, a highly expressed lncRNA in prostate cancer cells that can co-regulate Twist with miR-186 and promote tumor invasion, migration, and the progress of EMT [18]. This lncRNA/miRNA/mRNA pathway has been proposed as a new model by which lncRNAs regulate target gene expression through miRNA response elements [19]. Salmena et al. have termed this phenomenon as the competing endogenous RNA (ceRNA) theory [20].

In this study, we identified a HOXD-AS1/miR-186-5p/ PIK3R3 pathway in EOC as another example exhibiting the ceRNA property. The HOXD-AS1/miR-186-5p/ PIK3R3 pathway plays an important role in invasion, migration and EMT of EOC.

\section{Methods}

\section{Patients and EOC tissue samples}

We collected EOC $(n=36)$ and normal ovarian $(n=14)$ tissue samples from patients who underwent surgery at the Hunan Cancer Hospital (Affiliated Cancer Hospital of Xiangya School of Medicine, Central South University, Changsha, Hunan, China) from 2012 to 2014. The resected tissues were separately identified and confirmed by two pathologists and kept in liquid nitrogen immediately for subsequent use. In addition we also collected formalin-fixed paraffin-embedded (FFPE) specimens from an independent cohort of 200 patients to analyze the relationship of PFS and OS with the expression levels of HOXD-AS1. The clinicopathologic characteristics of patients are shown in Table 1 . The ethics committee of Hunan Cancer Hospital approved the protocol, and all patients signed informed consents before sample collection.

\section{Differentially expressed gene analysis}

Differentially expressed genes and lncRNAs were identified using the threshold of fold change $\geq 2$ and Student's t-test $p$-value $<0.01$ between EOC tumor versus normal tissues. The $\mathrm{R}$ package, Significance analysis of microarray (SAM) was used to identify differentially expressed
Table 1 Top ten significantly up-regulated and down-regulated IncRNAs in EOC

\begin{tabular}{|c|c|c|c|}
\hline \multicolumn{4}{|c|}{ Top ten up-regulated IncRNAs } \\
\hline & IncRNA ID & FC & $p$ \\
\hline 1 & CTD-2116 N17.1 & 54 & 0.001 \\
\hline 2 & MAL2 & 50 & 0.006 \\
\hline 3 & LINC00707 & 19 & 0.005 \\
\hline 4 & CXADRP3 & 18 & 0.001 \\
\hline 5 & HOXD-AS1 & 18 & 0.007 \\
\hline 6 & CDKN2B-AS1 & 16 & 0.004 \\
\hline 7 & ALDH3B1 & 15 & 0.000 \\
\hline 8 & XXbac-BPG254F23.6 & 11 & 0.004 \\
\hline 9 & $\mathrm{HCP} 5$ & 10 & 0.004 \\
\hline 10 & LINC00152 & 9 & 0.000 \\
\hline \multicolumn{4}{|c|}{ Top ten down-regulated IncRNAs } \\
\hline & IncRNA ID & FC & $p$ \\
\hline 1 & LINC00473 & -132 & 0.000 \\
\hline 2 & PWRN1 & -85 & 0.004 \\
\hline 3 & TRHDE-AS1 & -38 & 0.000 \\
\hline 4 & ADAMTS9-AS2 & -33 & 0.000 \\
\hline 5 & LINC00478 & -28 & 0.002 \\
\hline 6 & CECR7 & -28 & 0.000 \\
\hline 7 & СTC-454 M9.1 & -26 & 0.000 \\
\hline 8 & ADAMTS9-AS1 & -26 & 0.007 \\
\hline 9 & NR2F2-AS1 & -22 & 0.006 \\
\hline 10 & AL132709.5 & -22 & 0.007 \\
\hline
\end{tabular}

miRNA at a threshold of fold change $\geq 1.5$, false discovery rate (FDR) $q$-value $<0.05$. Hierarchical clustering was performed on the pheatmap package of $\mathrm{R}$ language and heatmap was constructed. Volcano maps were drawn using the ggplot2 packages.

\section{Gene ontology (GO) and Kyoto encyclopedia of genes} and genomes (KEGG) analysis

DAVID (The Database for Annotation, Visualization and Integrated Discovery, https://david.ncifcrf.gov/) was used to annotate the function of differentially expressed mRNA and enrichment of the signaling pathway. GO included cell component, biological process, and molecular function. Aberrant mRNAs were enriched into different pathways according to the KEGG database. TOP 10 terms were presented. The recommended $p$-value cutoff of $p<0.05$ was used.

\section{Target gene prediction}

The potential miRNAs that may bind to HOXD-AS1 were predicted by DIANA (http://carolina.imis.athena-innovation.gr/diana_tools/web/index.php? $r=$ lncbasev2\%2Findex-predicted). The candidate mRNA that 
may bind to miR-186-5p were predicted by starBase v2.0 (http://starbase.sysu.edu.cn/) database, which integrates five programs: targetScan, picTar, RNA22, PITA, and miRanda/mirSVR. Candidate mRNA are defined as the genes which are predicted to be target mRNA of miR-186-5p by at least three programs.

\section{Cell culture}

EOC cell line A2780 was cultured in Dulbecco's modified Eagle's medium (TransGen, Beijing, China), and EOC cell line SKOV3 and normal ovarian cell line IOSE80 were cultured in RPMI-1640 (TransGen, Beijing, China) with $10 \%$ fetal bovine serum (Gibco) and $1 \%$ penicillin/streptomycin (TransGen, Beijing, China). All of the cells were cultured in an incubator at $37^{\circ} \mathrm{C}$ with $5 \% \mathrm{CO}_{2}$.

\section{RNA extraction and RT-qPCR}

Total RNAs from tissues and cells were extracted using Trizol reagent (Invitrogen). Total RNAs from FFPE were extracted using RNeasy ${ }^{\circ} \mathrm{FFPE}$ Kit (Qiagen, Germany). cDNA was synthesized with the TransScript ${ }^{\circ}$ II One-Step gDNA Removal and cDNA Synthesis SuperMix Kit (TransGen, Beijing, China) according to the manufacturer's instructions. RT-qPCR was performed on the LightCycler 96 system (Roche) using a standard protocol from the TransStart $^{\circ}$ Tip Green qPCRSuperMix Kit (TransGen, Beijing, China). The results were normalized to GAPDH. The sequences of primers used for RT-qPCR assays are listed in Additional file 1.

For the detection of miRNA expression, reverse transcription and qPCR were performed using stem-loop primers and Bulge-Loop ${ }^{\text {tm }}$ miRNA RT-qPCR Starter Kit from Ribobio (Guangzhou, China). U6 snoRNA was used as the endogenous control. All experiments were performed in triplicate according to the manufacturer's manual. Expression fold changes were calculated using $2^{-\Delta \Delta C t}$ methods.

\section{In situ hybridization (ISH)}

In situ hybridization was performed to detect the expression of HOXD-AS1, miR-186-5p, and PIK3R3. The probes of HOXD-AS1, miR-186-5p, and PIK3R3 were designed by BOSTER (Wuhan, China). RNA ISH was performed using the in situ hybridization detection kit (BOSTER) and DAB chromogenic kit (ZSGB-BIO) on $3-\mu \mathrm{m}$ FFPE tissue sections according to the manufacturer's instructions. The staining scores were determined based on both the intensity and proportion of target gene-positive cells under 20X and 40X objective.

\section{Small interfering RNA (siRNA) and miRNA inhibitor transfections}

LncRNA HOXD-AS1, siRNA (si-HOXD-AS1), PIK3R3 siRNA (si-PIK3R3), miR-186-5p inhibitor, and negative control (si-NC, miR-NC) were all obtained from Ribobio (Guangzhou, China). RiboFECT ${ }^{\text {max }}$ CP transfection Kit (Guangzhou, China) was used for cell transfection based on manufacturer's instructions. SKOV3 and A2780 were seeded in a six-well plate and transfection would be performed when cell's confluence reached $50 \%$. Cells were transfected with a final concentration of $100 \mathrm{nM}$ of individual siRNA and $200 \mathrm{nM}$ of miR-186-5p inhibitor. All of the steps were carried out according to the manufacturer's specification. Experiments were performed three times.

\section{Wound healing assay}

The cell monolayer was scraped in straight lines using a 10ul pipette tip and washed with PBS two times when transfected cells were up to $80 \%$ confluence in a six-well culture dish. The cells were cultured in culture medium with $3 \%$ FBS and $1 \%$ penicillin/streptomycin (TransGen, Beijing, China). Images were captured at $0,12,24,36$, and $48 \mathrm{~h}$ following the initial scratch from five different areas for each wound. We used Image J (National Institutes of Health, Bethesda, MD, USA) software to calculate cell wound healing rate as: (the original wound areas - the actual wound areas at different times)/ (the original wound areas).

\section{Trans-well assays}

The invasion ability was measured using transwell chambers coated with 1:30 diluted Matrigel Basement Membrane Matrix (Corning Life Sciences, NY, USA). Migration assays were performed without matrigel. $1 \times 10^{5}$ cells were seeded into upper chambers $(8 \mu \mathrm{M}$ pore size, Corning Life Sciences, NY, USA) with 200ul serum-free media. A total of $600 \mathrm{ul}$ of $20 \%$ FBS media was added into the lower chambers. After incubation for $48 \mathrm{~h}$ at $37^{\circ} \mathrm{C}$, the cells in the upper chamber that did not invade through the pores were wiped out with cotton wool. The cells in the lower chamber were fixed with methanol and stained with $0.5 \%$ crystal violet. Cells were counted and imaged at $\times 200$ magnification. The experiments were repeated three separate times.

\section{Western blotting}

After transfection for $48 \mathrm{~h}$, cells were lysed using RIPA buffer (Thermo, USA) with proteinase inhibitor cocktail (Roche), then incubated at $4{ }^{\circ} \mathrm{C}$ for $15 \mathrm{~min}$. The lysate was centrifuged and protein concentration was measured by BCA Protein Assay Kit (Thermo, USA). The protein was separated on $10 \%$ sodium dodecyl sulfatepolyacrylamide gel electrophoresis (SDS-PAGE) and then transferred to $0.22 \mu \mathrm{m}$ PVDF membrane (Thermo, USA). After blocking with $5 \%$ fat-free milk for $1 \mathrm{~h}$, the membranes were incubated with primary antibodies at $4{ }^{\circ} \mathrm{C}$ overnight with gentle shaking. Next, the membranes were incubated with secondary antibody at room temperature for $2 \mathrm{~h}$. Finally, the immunofluorescence of protein bands was visualized using Pierce ${ }^{\text {rax }}$ ECL Western Blotting Substrate $(32,109$, 


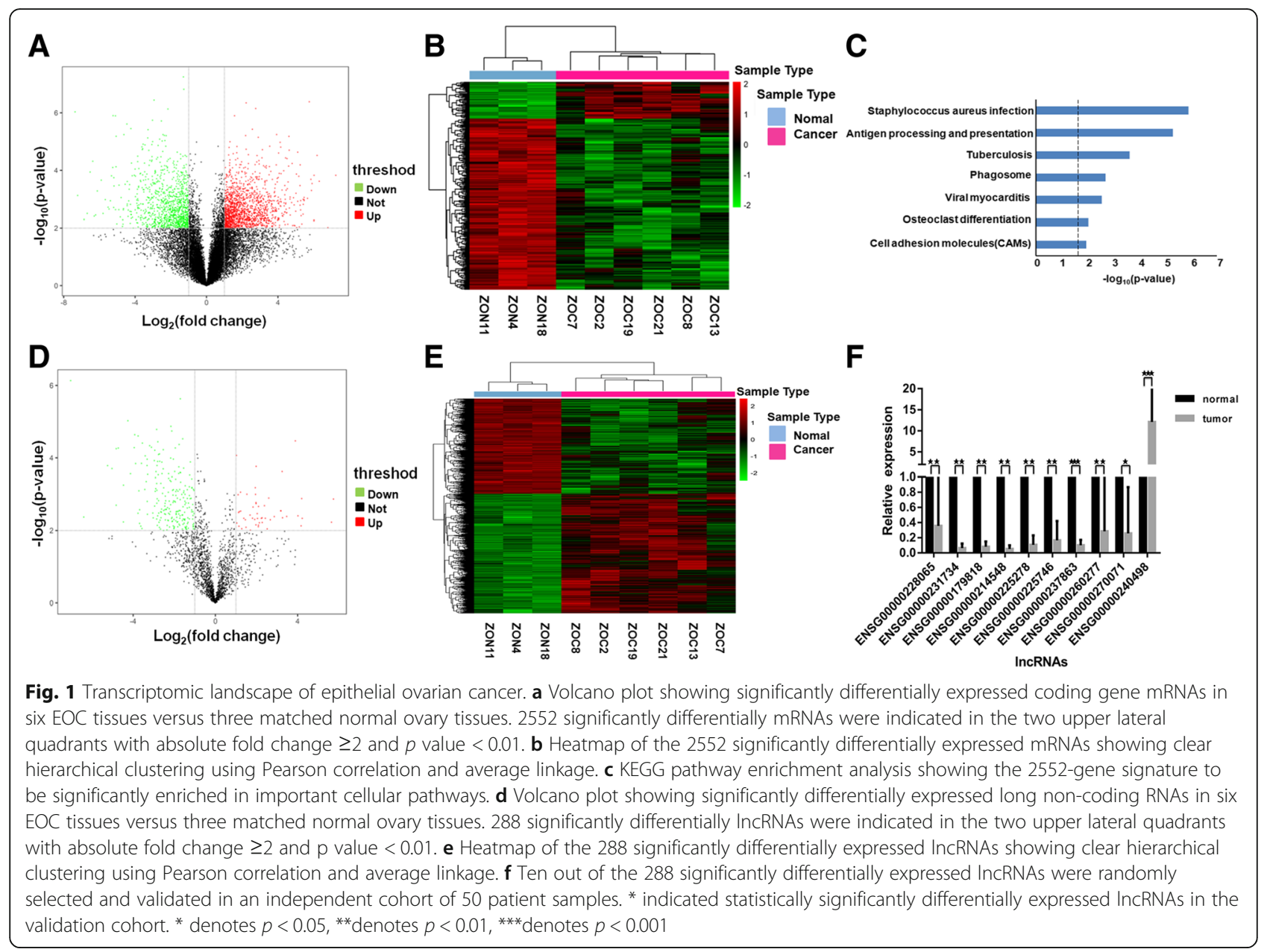

Thermo Scientific, USA) by Image J 2 software (Madison, WI, USA). Primary antibodies are as following: E-cadherin (1: 500, ab15148, Abcam company), Vimentin (1:500, \#7431, Cell Signaling Technology (CST)), GAPDH (1:5000, 60,004-1-lg, Proteintech company), Rac1 (1:500, ab33186, Abcam), Twist (1500, GTX60776, Gene Tex).

\section{Dual-luciferase reporter assay}

The dual luciferase reporter plasmids (LncRNA-HOXD-AS1-WT, LncRNA-HOXD-AS1-Mut, PIK3R3-WT, PIK3R3-Mut, miR-186-5p, and negative control) were synthesized by GenePharma (Shanghai, China). The SKOV3 cells were seeded into 96-well plates and
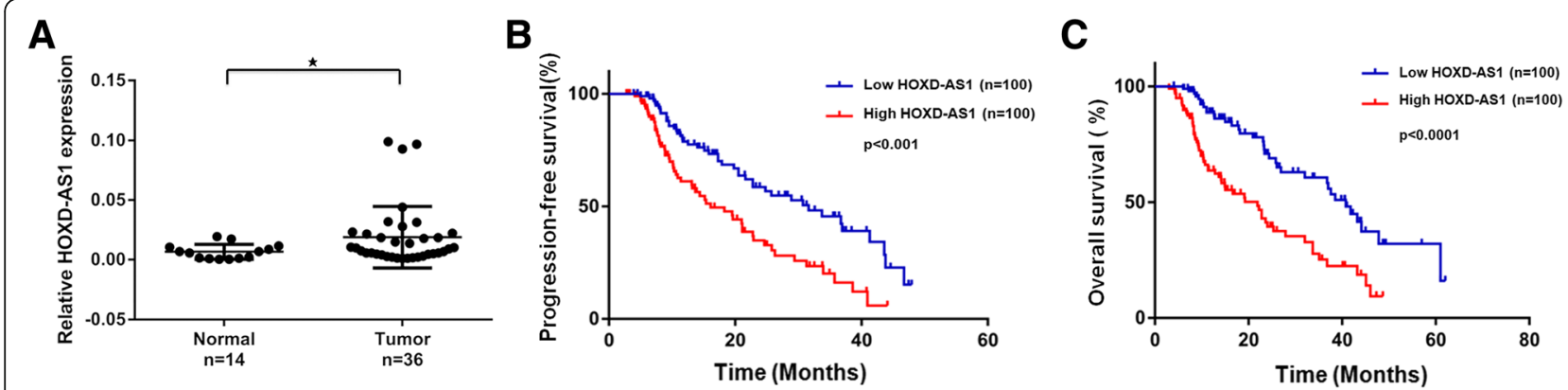

Fig. 2 HOXD-AS1 is up-regulated in EOC tissues and predicts poor prognosis. a Relative expression of HOXD-AS1 measured using RT-qPCR and normalized against GADPH in EOC tissues $(n=36)$ and normal ovarian tissues $(n=14)$. Data were expressed as means \pm SD. Kaplan-Meier survival analysis showing high HOXD-AS1 expression (above median expression) was significantly associated with poorer progression-free survival (b) and overall survival (c) of EOC patients $(n=200)$. ${ }^{\star}$ denotes $p<0.05,{ }^{\star \star}$ denotes $p<0.01$ 
Table 2 Relationship between HOXD-AS1 expression and clinicopathologic of EOC patients

\begin{tabular}{|c|c|c|c|c|c|}
\hline \multirow[t]{2}{*}{ Characteristics } & \multirow{2}{*}{$\begin{array}{l}\text { Number } \\
\text { of cases }\end{array}$} & \multicolumn{2}{|l|}{ HOXD-AS1 } & \multirow[t]{2}{*}{$p$ value } & \\
\hline & & Low $(n=100)$ & $\operatorname{High}(n=100)$ & & \\
\hline Age (years) & & & & 0.479 & \\
\hline$<50$ & 95 & 50 & 45 & & \\
\hline$\geq 50$ & 105 & 50 & 55 & & \\
\hline Histological subtype & & & & 0.635 & \\
\hline Serous & 145 & 71 & 74 & & \\
\hline Other & 55 & 29 & 26 & & \\
\hline Grade & & & & 0.002 & ** \\
\hline G1 & 82 & 52 & 30 & & \\
\hline G2/G3 & 118 & 48 & 70 & & \\
\hline FIGO stage & & & & 0.002 & ** \\
\hline$|/| \mid$ & 46 & 32 & 14 & & \\
\hline III/IV & 154 & 68 & 86 & & \\
\hline Lymph node metastasis & & & & 0.011 & $*$ \\
\hline Negative & 133 & 75 & 58 & & \\
\hline Positive & 67 & 25 & 42 & & \\
\hline Serum CA125 & & & & 0.064 & \\
\hline$<500$ & 113 & 50 & 63 & & \\
\hline$\geq 500$ & 87 & 50 & 37 & & \\
\hline Ascites & & & & 0.396 & \\
\hline Absent & 100 & 53 & 47 & & \\
\hline Present & 100 & 47 & 53 & & \\
\hline
\end{tabular}

FIGO International Federation of Gynecology and Obstetrics

cultured to $80 \%$ confluence. The plasmid vectors or empty plasmid were transiently transfected into SKOV3 cells using lipofectamine 3000 (Invitrogen). After $48 \mathrm{~h}$ incubation, the luciferase activity was examined by Dual-Glo ${ }^{\circ}$ Luciferase Assay System (Promega, USA) according to the manufacturer's protocol.

\section{Statistical analysis}

All experiments were performed three separate times. Statistical analysis was performed with SPSS 13.0 and graphs were constructed with GraphPad Prism 5 (La Jolla, CA, USA). The data were presented as the mean \pm Standard Deviation (SD). The significance of differences between groups

Table 3 Univariate and multivariate analysis ${ }^{\mathrm{a}}$ of clinicopathological parameters in association with overall survival ${ }^{\mathrm{b}}$

\begin{tabular}{|c|c|c|c|c|c|c|c|c|}
\hline \multirow[t]{2}{*}{ Variable } & \multicolumn{4}{|c|}{ Univariable analysis } & \multicolumn{4}{|c|}{ Multivariate analysis } \\
\hline & $\mathrm{HR}$ & $95 \% \mathrm{Cl}$ & $p$ value & & $\mathrm{HR}$ & $95 \% \mathrm{Cl}$ & $p$ value & \\
\hline Age & 0.82 & $0.54-1.25$ & 0.352 & & & & & \\
\hline Histological subtype & 0.82 & $0.50-1.35$ & 0.44 & & & & & \\
\hline Grade & 2.02 & $1.19-3.41$ & 0.009 & * & 1.88 & $1.13-3.13$ & 0.015 & * \\
\hline FIGO stage & 2.63 & $1.29-5.34$ & 0.008 & * & 2.27 & $1.16-4.46$ & 0.017 & * \\
\hline Lymph node metastasis & 1.92 & $1.19-3.10$ & 0.008 & * & 1.95 & $1.20-3.15$ & 0.007 & * \\
\hline Serum CA125 & 0.98 & $0.62-1.55$ & 0.925 & & & & & \\
\hline Ascites & 0.70 & $0.45-1.08$ & 0.11 & & & & & \\
\hline HOXD-AS1 expression & 1.94 & $1.22-3.06$ & 0.005 & * & 1.92 & $1.23-3.00$ & 0.004 & * \\
\hline
\end{tabular}

${ }^{\mathrm{a} C o x}$ hazard model

b200 patients with full clinical information including age, FIGO stage,histological type,type of chemotherapy and type of surgery.HR,hazard ratio;Cl,confidence interval;FIGO, International Federation of Gynecology and Obstetrics; CA125, carbohydrate antigen 125; HOXD-AS1, HOXD Cluster Antisense RNA 1 
was assessed by Student's t-test and Chi-square test. The Kaplan-Meier method test was used for PFS analysis and OS analysis. Pearson correlation analysis was used to determine the relationship between two genes. We used the univariate and multivariate Cox proportional hazards modeling to determine the effects of variables on survival. A $p$-value of $<0.05$ was considered statistically significant.

\section{Results}

\section{Transcriptome landscape of EOC}

We performed transcriptome profiling on 6 human epithelial ovarian cancer tissues and three matched normal ovary samples using the Agilent Human IncRNArray + mRNA Array V4.0. at a threshold of absolute fold change of greater than 2 and p-value of less than 0.01 . We observed a total of 2552 mRNAs in EOC to be differentially expressed compared to normal ovary samples, of which 1496 were up-regulated, and 1056 were down-regulated (Fig. 1a and b). These differentially expressed mRNAs were enriched in a number of KEGG pathways such as Straphylococcus aureus infection and cell adhesion molecules which are relevant pathways linking EOC for either increased risk or aggressiveness of the cancer (Fig. 1c). In the 1922 well-annotated lncRNAs on the array, we observed 288 lncRNAs to be differentially expressed (Fig. 1d). Among which, 51 were significantly up-regulated and 237 were significantly down-regulated in EOC tumors versus the normal ovary tissues. These 288 lncRNAs can stratify the samples into distinct clusters based on expression values (Fig. 1e). We validated the robustness of the array data by randomly choosing ten lncRNAs from these 288 differentially

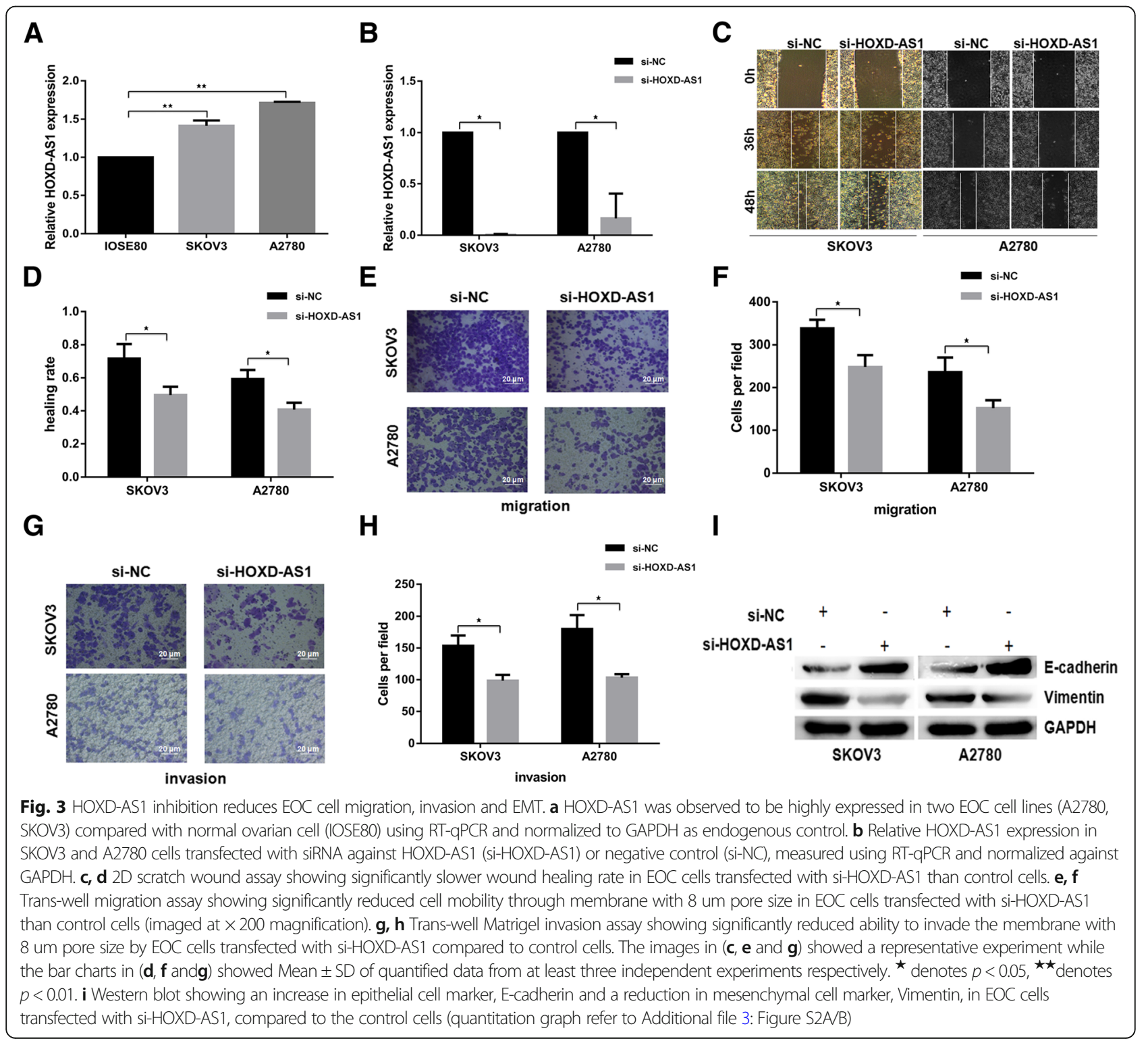


expressed lncRNAs in an independent cohort of 50 samples comprising of 36 EOC tissues and 14 normal ovary tissues. All ten lncRNAs showed differential expression between EOC and normal ovary tissues which was in concordance with what we observed in the microarrays. In conclusion, the transcriptome profile constructed from high quality data using the Agilent Human lncRNArray + mRNA Array were reproducible in an independent cohort. Table 1 summarized the top ten most up-regulated and down-regulated lncRNAs in EOC tumors and we selected HOXD-AS1 for further characterization.

\section{HOXD-AS1 is highly expressed in EOC tissues which negatively impacts patients survival}

We performed RT-qPCR to validate HOXD-AS1 up-regulation in an independent cohort comprising of $36 \mathrm{EOC}$ tissues and 14 normal ovarian tissues and found HOXD-AS1 to be consistently and significantly up-regulated in EOC tissues ( $p$ $<0.05$, Fig. 2a). We further examined HOXD-AS1 expression in another independent cohort of 200 EOC FFPE samples and correlated HOXD-AS1 expression with the clinicopathological features of EOC patients. As shown in Table 2, high HOXD-AS1 expression (above the median value $=0.000565$ ) was positively associated more aggressive EOC tumors characterized by higher tumor grade (Grade G2/3), advanced stage (FIGO stage III/IV), and the presence of lymph node metastasis $(\mathrm{p}<0.05)$. We observed that high HOXD-AS1 level was significantly associated with poorer PFS and OS of EOC patients through Kaplan-Meier analysis $(p<0.05$, Fig. $2 b$ and c). Univariate Cox regression analysis showed that the poor prognosis was observed in EOC patients with high-grade tumor, FIGO stage III / IV, lymph node

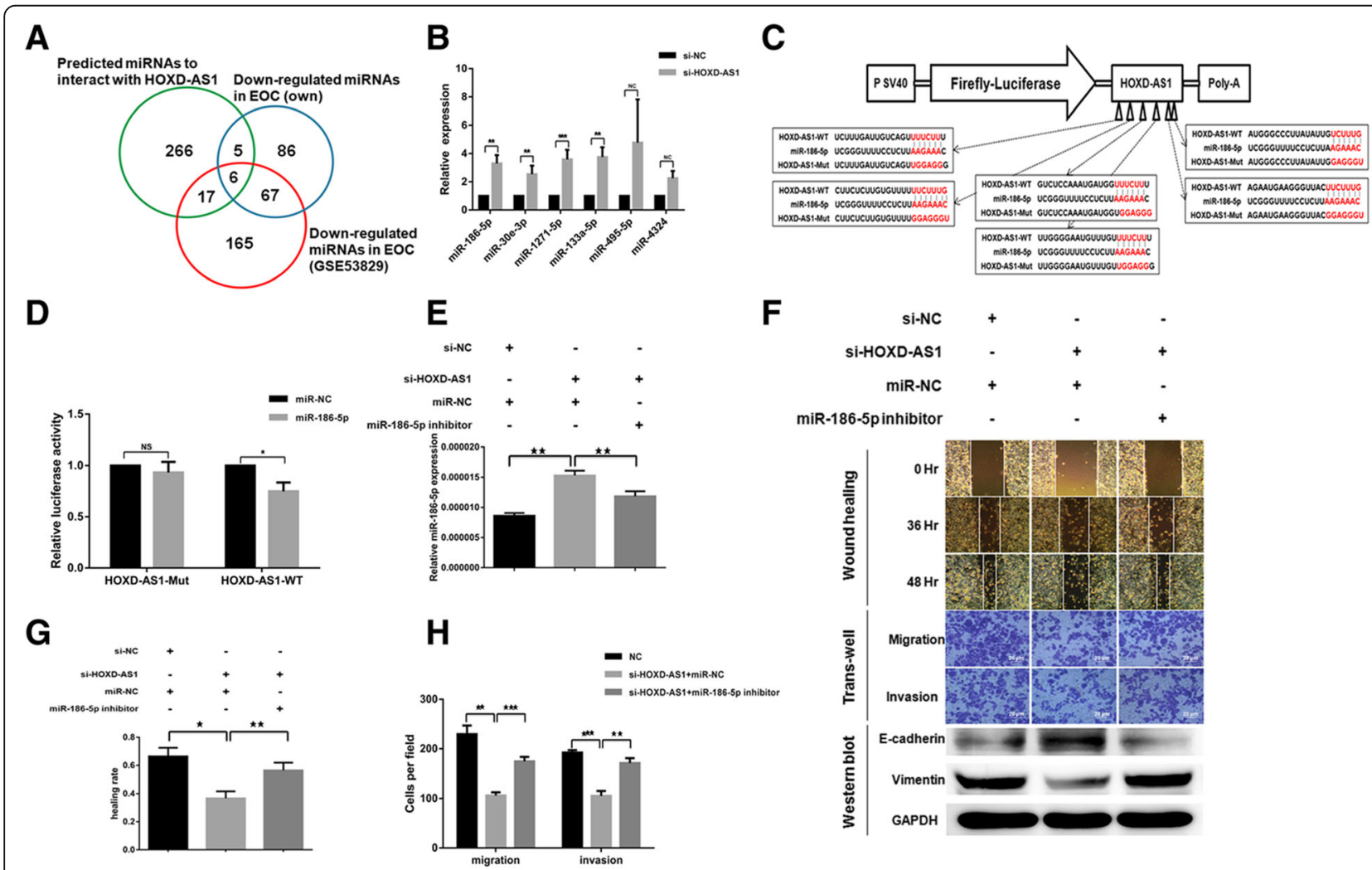

Fig. 4 HOXD-AS1 binds to miR-186-5p to promote EOC migration, invasion and EMT. a Venn diagram showing the six overlapped miRNAs which were significantly down-regulated in the Affymetrix miRNA arrays and GSE53829, and predicted to be a target of HOXD-AS1. b Relative expression of the six miRNAs in (a) in EOC cells transfected with siRNA against HOXD-AS1 or si-NC control, measured using RT-qPCR. c Schema showing the cloning of wild type as well as a mutant HOXD-AS1 in which all six putative miR-186-5p binding sites were mutated in a luciferase reporter construct. $\mathbf{d}$ Direct interaction of HOXD-AS1 and miR-186-5p on the putative miR-186-5p binding sites as shown by the specific reduction of relative luciferase reporter activities in cells co-transfected with miR-186-5p and wild type HOXD-AS1 reporter construct. e miR-186$5 p$ expression was elevated in EOC cells transfected siRNA against HOXD-AS1 and this could be reversed by the co-transfection of miR-186-5p inhibitors, as shown in RT-qPCR with U6 snoRNA normalization. ${ }^{\star}$ denotes $p<0.05,{ }^{\star \star}$ denotes $p<0.01 . \mathbf{f}$ EOC cells transfected with si-HOXD-AS1 showed reduced ability to migrate and invade in vitro as measured through wound healing and trans-well assays (imaged at $\times 200$ magnification) than control cells, with a corresponding increase in E-cadherin (an epithelial marker) and decrease in Vimentin (a mesenchymal marker). These changes in phenotypes could be partially rescued with the co-transfection of miR-186-5p inhibitors which partially reversed the miR-186-5p elevation by HOXD-AS1 knock-down, suggesting HOXD-AS1 mediated these phenotypic changes through miR-186-5p (quantitation graph of WB refer to Additional file 3: Figure S2C). g, h Relative wound healing rate $(\mathbf{g})$ and transwell migration/invasion(h) from at least three independent experiments as described in(f) 
metastasis, and high HOXD-AS1 expression. Multivariate Cox regression analysis suggested that tumor grade, FIGO stage, lymph node metastasis status, and HOXD-AS1 expression are independent risk predictors of prognosis for EOC patients (Table 3). Our data suggested that HOXD-AS1 may play an important functional role in EOC tumor.

\section{HOXD-AS1 promotes EOC cell migration, invasion, and EMT transition}

To understand the molecular function of HOXD-AS1 on EOC cells, we designed HOXD-AS1-specific siRNAs to inhibit the endogenous HOXD-AS1 expression in SKOV3 and A2780 EOC cell lines, both of which showed higher HOXD-AS1 expression than normal ovarian cell line IOSE80 (Fig. 3a). SKOV3 and A2780 cells showed significantly reduced ability to migrate in wound healing assays (Fig. 3c and d) when HOXD-AS1 was knocked-down (Fig. 3b). EOC cells in trans-well assays transfected with siRNA against HOXD-AS1 consistently demonstrated significantly reduced capacity to migrate or invade when compared to the mock-transfected control cells (Fig. $3 e-h)$. EMT process is closely related to the invasion and migration of cancer cells therefore we examined the EMT markers between the HOXD-AS1 knocked-down cells and control cells. As shown in Fig. 3i, we observed a significant increase in the expression of epithelial marker E-cadherin and a corresponding decrease in mesenchymal marker vimentin in the HOXD-AS1 knock-down cells as compared to controlls. These results suggested that the down-regulation of HOXD-AS1 inhibited the invasion, migration, and EMT process of EOC cells in vitro.

\section{HOXD-AS1 binds miR-186-5p to promote cell migration, invasion, and EMT}

We performed Affymetrix miRNA 4.0 chips to evaluate if HOXD-AS1 can increases EOC cell migration, invasion, and EMT through its interaction with miRNAs in a ceRNA manner. We identified a total of 164 miRNAs to be significantly down-regulated in the same nine samples profiled for miRNAs of which 73 were found to be consistently reported by an independent GEO dataset (GSE53829). We further shortlisted a total of 6 miRNAs by overlapping these 73 down-regulated miRNAs in EOC tissues with 293 miRNAs predicted to interact with HOXD-AS1 by DIANA algorithm (Fig. 4a and Table 4). The increased miR-186-5p expression amongst these 6 miRNAs were confirmed upon HOXD-AS1 inhibition (Fig. 4b). We selected miR-186-5p for further research because miR-186-5p consistently showed an inverse correlation with HOXD-AS1 in both patient samples and cell lines. HOXD-AS1 with 6 putative miR-186-5p binding sites further enforced our interest. Inhibition of miR-186-5p promote cell migration, invasion and EMT in EOC cells (Additional file 2: Figure S1).

To demonstrate that HOXD-AS1 interacts with miR-186-5p through its putative miR-186-5p binding sites, we cloned the wildtype and a mutant HOXD-AS1 in which all six putative miR-186-5p binding sites were mutated and inserted downstream of a firefly luciferase gene (Fig. 4c). As shown in Fig. 4d, we observed significantly reduced reporter activity in the wildtype HOXDAS1 construct when the cells were co-transfected with miR-186-5p compared to wildtype HOXD-AS1 construct co-transfected with the miRNA controls. However, such difference was abrogated when the putative miR-186-5p binding sites were mutated, indicating that HOXD-AS1 physically interacts with miR-186-5p at its putative binding sites to regulate reporter gene activity. It is further evidenced by concurrent increase in miR186-5p expression when EOC cells were transfected with siRNAs targeting HOXD-AS1. The HOXD-AS1 knockeddown cells exhibited more epithelial and less mesenchymal phenotype (Fig. 4e, f, g and h, middle panel) which lead to reduced ability to migrate or invade. We observed a corresponding reversal of the above phenotype in cell migration, invasion, and EMT (Fig. 4e and f right panel) when miR-186-5p inhibitors were co-transfected with si-HOXD-AS1 to partly negate the increase in miR186-5p expression. Therefore, our data demonstrated

Table 4 List of potentially HOXD-AS1 regulated miRNAs

\begin{tabular}{|c|c|c|c|c|c|c|c|c|}
\hline \multirow[t]{3}{*}{ miRNA ID } & \multicolumn{4}{|c|}{ miRNA expression in EOC tumors versus normal ovary tissues } & \multirow{3}{*}{$\begin{array}{l}\text { Targeting HOXD-AS1 in silico } \\
\text { LncBase Score }\end{array}$} & \multirow{2}{*}{\multicolumn{3}{|c|}{$\begin{array}{l}\text { miRNA changes upon } \\
\text { HOXD-AS1 inhibition }\end{array}$}} \\
\hline & \multicolumn{2}{|c|}{ Affymetrix 4.0} & \multicolumn{2}{|c|}{ GSE53829 } & & & & \\
\hline & $\overline{F C}$ & q-value & $\overline{F C}$ & q-value & & $\overline{F C}$ & $p$-value & \\
\hline hsa-miR-186-5p & -4.0 & 0.01 & -1.9 & 0.04 & 0.93 & 3.3 & 0.01 & ** \\
\hline hsa-miR-30e-3p & -5.0 & 0.03 & -1.8 & 0.02 & 0.77 & 2.5 & 0.00 & ** \\
\hline hsa-miR-1271-5p & -6.5 & 0.00 & -6.0 & 0.00 & 0.76 & 3.5 & 0.00 & *** \\
\hline hsa-miR-133a-3p & -10.1 & 0.00 & -2.2 & 0.12 & 0.99 & 3.7 & 0.01 & $* *$ \\
\hline hsa-miR-495-3p & -15.7 & 0.01 & -3.2 & 0.01 & 0.87 & 3.6 & 0.42 & NS \\
\hline hsa-miR-4324 & -24.1 & 0.00 & -3.0 & 0.00 & 0.87 & 2.3 & 0.18 & NS \\
\hline
\end{tabular}

FC fold change, q-value, FDR q value; NS not statistically significant 
HOXD-AS1 promotes cell migration, invasion, and EMT through inhibiting miR-186-5p.

\section{miR-186-5p targets PIK3R3 to negatively regulate cell migration, invasion, and EMT}

In order to investigate how miRNAs regulate cellular functions through its target genes we queried starBase v2.0 to identify a total of 284 predicted targets of miR-186-5p, among which 33 were significantly up-regulated in EOC tissues with low miR-186-5p expression (Fig. 5a). KEGG pathway enrichment analysis identified four pathways such as focal adhesion all are important for cell migration and invasion. PIK3R3 was involved in all four pathways which suggestes PIK3R3 might be a direct miR-186-5p target. To test this hypothesis, we cloned the wildtype 3 ' untranslated region (3'UTR) of PIK3R3 and inserted into the

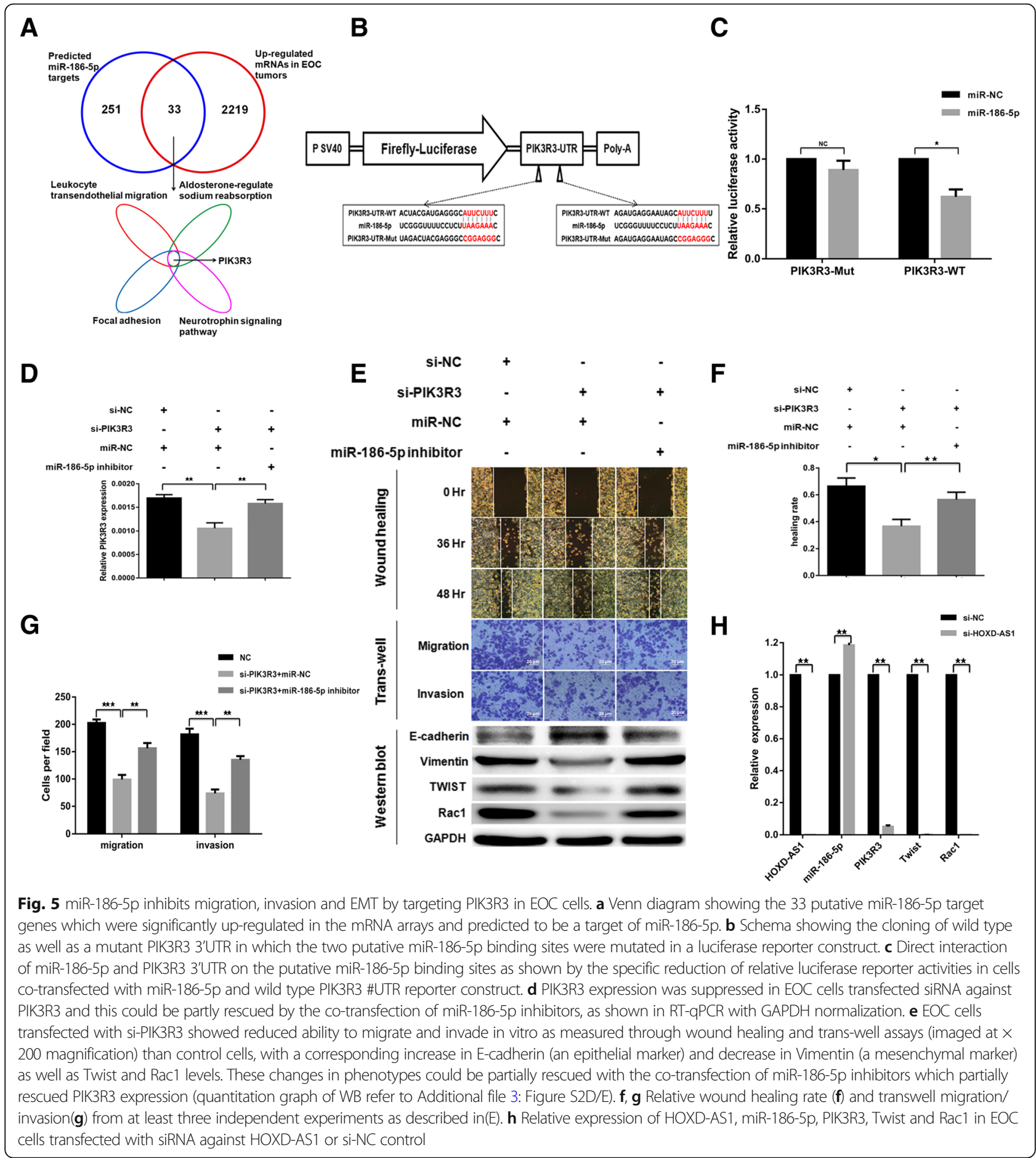




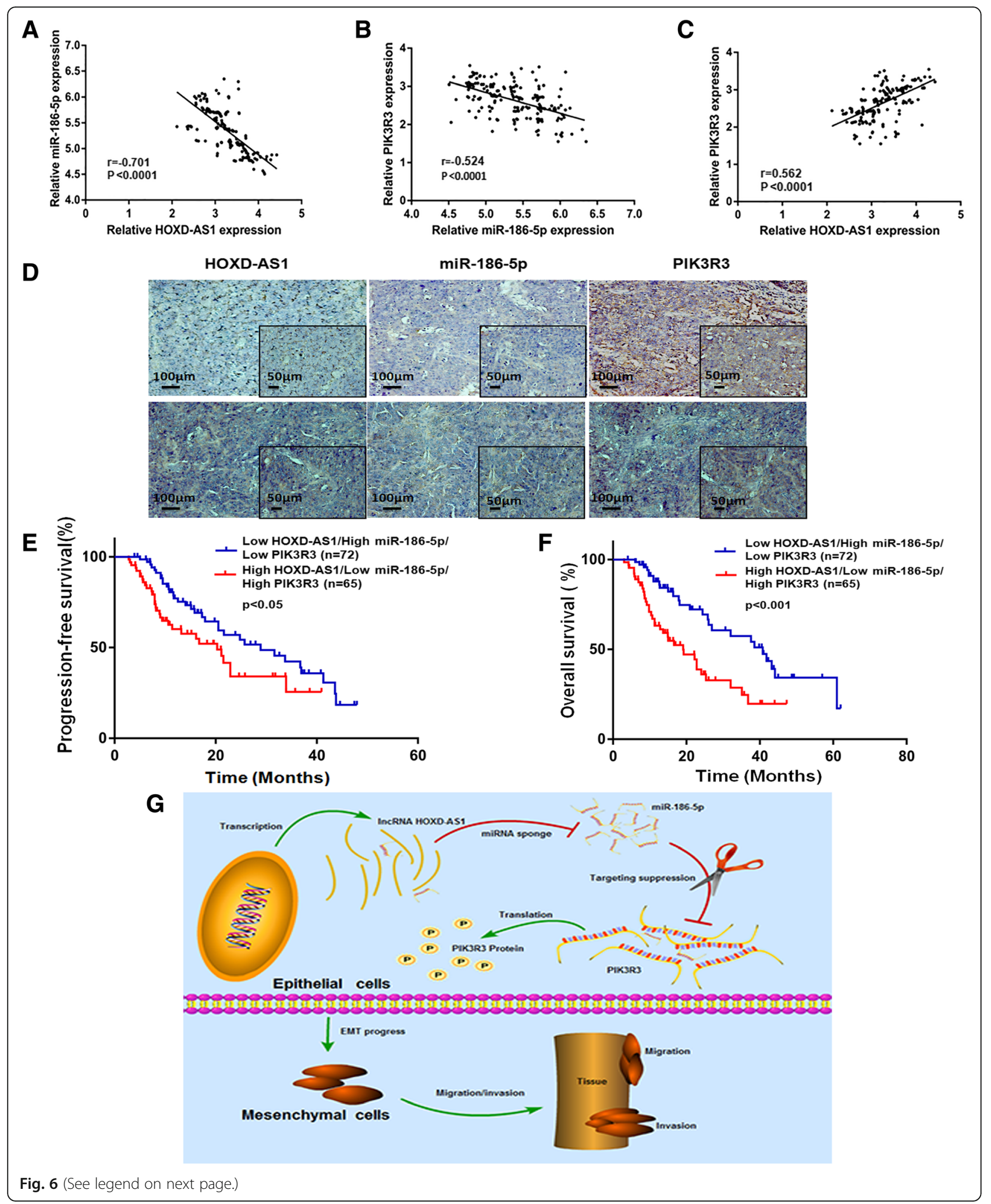


(See figure on previous page.)

Fig. 6 HOXD-AS1/miR-186-5p/PIK3R3 is a clinically relevant pathway. a Pearson correlation showing significant inverse correlation between HOXD-AS1 and miR-186-5p in 200 EOC tissues. b Pearson correlation showing significant inverse correlation between miR-186-5p and PIK3R3 in 200 EOC tissues. c Pearson correlation showing significant positive correlation between HOXD-AS1 and PIK3R3 in 200 EOC tissues. d RNA ISH assay of HOXD-AS1, miR-186-5p, PIK3R3 in HOXD-AS1 high expression ovarian cancer sample and HOXD-AS1 low expression ovarian cancer sample. e, $\mathbf{f}$ Kaplan Meier survival curve showing significantly poorer progression-free $(\mathbf{e})$ and overall (f) survival in patients who showed high HOXD-AS1, low miR-186-5p and high PIK3R3 compared to patients who showed the reciprocal expression of these three factors. $\mathbf{g}$ Diagram summarizing the mechanism of action of HOXD-AS1/miR-186-5p/PIK3R3 on EMT in EOC cells

downstream region of the luciferase reporter gene. We mutated the two putative miR-186-5p binding sites along the PIK3R3 3'UTR to construct a mutant clone (Fig. 5b). Specific reduction in luciferase activity was only observed in EOC cells co-transfected with miR-186-5p and wildtype PIK3R3 3'UTR but not the mutant PIK3R3 3'UTR, confirming our hypothesis that miR-186-5p interacts with putative binding sites along PIK3R3 3'UTR to down-regulated luciferase reporter gene expression (Fig. 5c). Furthermore, EOC cells transfected with siRNA against PIK3R3 exhibited greater epithelial phenotypes and reduced ability to migrate and invade. This effect could be rescued by the co-transfection of miR-186-5p inhibitors as our data suggested that miR-186-5p binds to PIK3R3 to negatively regulate cell migration, invasion, and EMT (Fig. 5d, e, f \& g). As shown in Fig. 5h, in EOC cells transfected with siRNA against HOXD-AS1, we observed increased miR-186-5p and decreased PIK3R3 along with Twist and Rac1 which contributed to reduced cell migration, invasion and EMT.

\section{HOXD-AS1/miR-186-5p/PIK3R3 is a clinically relevant pathway regulating EMT in EOC tissues}

We proceeded to evaluate the clinical relevance of the in vitro HOXD-AS1/miR-186-5p/PIK3R3 pathway in 200 FFPE EOC samples. We observed significant inverse correlations between HOXD-AS1/miR-186-5-p (Fig. 6a) and between miR-186-5p/PIK3R3 (Fig. 6b) and significant positive correlation between HOXD-AS1 and PIK3R3 (Fig. 6c) using RT-qPCR quantitation. Additional in situ hybridization (ISH) data were shown to be consistent with RT-qPCR data (Fig. 6d). Furthermore, patients with high HOXD-AS1 (above the median value $=0.000565$ ), low miR-186-5p (below the median value $=0.00000413$ ), and high PIK3R3 (above the median value $=0.001908$ ) expression showed significantly poorer PFS and OS compared to patients with low HOXD-AS1, high miR-186-5p, and low PIK3R3 (Fig. 6e and f). As summarized in Fig. $6 \mathrm{~g}$, in normal ovary cells, banlanced HOXD-AS1and miR-186-5p expression keeps EMT in check by regulating PIK3R3. In EOC cells, significantly elevated HOXD-AS1 level suppresses cellular miR-186-5p leading to increased PIK3R3 level which in turn leads to EMT.

\section{Discussion}

Tumor metastasis is a major factor contributing to the poor prognosis of EOC patients [21, 22]. EMT is a crucial cellular process associated with metastasis in tumor cells $[23,24]$. Our group has previously demonstrated that platinum-based drugs could induce the activation of Rac1 and initiate EMT [25]. Understanding the crucial players in the EMT process in EOC will provide insights into the development of new therapeutic strategies to treat the disease.

LncRNAs are an class of non-coding RNAs which plays an important regulatory role in the biological processes of tumorigenesis, tumor cell proliferation, invasion, and migration [15]. In EOC, aberrant expression of lncRNA regulates target genes through different mechanisms affecting biological behaviour of tumor cells. For example, lncRNA ABHD11-AS1 through regulating RhoC can promote A2780 and OVCAR3 proliferation and inhibit apoptosis [26]; IncRNA PTAR through miR-101 -3p/ZEB1 pathway behave in ceRNA model promotes invasion, migration, and EMT in EOC [27]; lncRNA PANDAR through SFRS2/p53 phosphorylation involved in the acquired Cisplatin resistance [28]. IncRNA aberrant expression in EOC can affect tumor proliferation, migration, and drug sensitivity. It also plays an important regulatory role in cancer initiation and progression.

lncRNAs have been divided into 5 categories based on the location of the lncRNA gene in the genome: (1) sense lncRNA, (2) antisense lncRNA, (3) bidirectional lncRNA, (4) intronic lncRNA, and (5) intergenic lncRNA [29]. In this study, we systematically investigated lncRNAs deregulation in EOC tissues versus normal ovary tissues and identified HOXD-AS1 to be significantly up-regulated in EOC tumors. HOXD-AS1 is an antisense lncRNA located on chromosome $2 \mathrm{q} 31.2$. Lu et al. have previously shown that HOXD-AS1 is over-expressed in metastatic hepatocellular carcinoma and promotes hepatocellular carcinoma metastasis through miR19a/ARHGAP11A signaling pathway [30]. Furthermore HOXD-AS1 has been demonstrated to cooperate with miR-130a to regulate SOX4 and E2F8, and promote metastasis in hepatocellular carcinoma and glioma respectively [31, 32]. In this study, we demonstrated that HOXD-AS1 acting as a cellular sponge in a ceRNA mechanism inhibits miR-186-5p and resulted in the corresponding up-regulation of PIK3R3 in EOC cells. 
This novel HOXD-AS1/miR-186-5p/PIK3R3 pathway plays an important role in EMT in EOC cells. In addition, high HOXD-AS1 level is significantly associated with poorer PFS and OS of EOC patients (Figs. 2 \& 6), and hence demonstrating the clinical relevance of this novel pathway.

MiRNA-186-5p can bind to HOXD-AS1 and affect the expression of downstream target genes in the form of ceRNA. Abnormal expression of miRNA-186-5p in various tumors plays a regulatory role in the process of EMT. For example, high expression of miR-186-5p in colorectal cancer cells can inhibit the proliferation, metastasis, and EMT process of tumor cells; Expression changes of miR-186-5p are closely related to the proliferation and invasion ability of non-small cell lung cancer [33]. Therefore, the study further focused on whether HOXD-AS1 can regulate the expression of miR-186-5p through a ceRNA sponge model. Data of the present study suggests that HOXD-AS1 can negatively regulate miR-186-5p.

PIK3R3 is a regulatory subunit of PI3K, whose differential expression can promote the proliferation, invasion, and migration process of NSCLC cells, cervical carcinoma cells, and pancreatic cancer cells. Our studies have suggested that PIK3R3 is the target gene regulated by HOXD-AS1/miRNA-186-5p in the mode of ceRNA. PIK3R3 was down-regulated in SKOV3 cells transfected with si-PIK3R3 accompanied with the down-regulation of Twist and Rac1, which suggests Twist and Rac1 may mediate changes in PIK3R3 expression and affecting EMT in tumor cells [34-36].

In this study, we used IncRNA, microRNA, and mRNA biochips as carriers to discover a number of differentially expressed genes in ovarian cancer tissues. We utilized GEO, KEGG, DIANA and other common tumor databases for bioinformatics analysis. The results suggest that HOXD-AS1, as a ceRNA, upregulates PIK3R3 expression by sponging miR-186-5p. The higher expression of HOXD-AS1 is associated with shorter PFS and OS of EOC patients. Collectively, lncRNA-HOXD-AS1 may function as a promising biomarker and therapeutic target for human EOC treatment.

While this paper is in the process of preparation, Wang et al. reported HOXD-AS1 could regulate cell migration and invasion through miR-608 and FZD4 in ovarian cancer [37]. Therefore, these data suggest that HOXD-AS1 may function as a master regulator of metastasis through its interaction with a number of miRNAs and their respective target genes. HOXD-AS1 functions as a cellular sponge to modulate the availability of specific miRNAs at the 3'UTR of the miRNA target genes in ceRNA theory [20].

\section{Conclusions}

In summary, we profiled the transcriptome landscape to report significant differentially expressed mRNA, IncRNA and miRNAs in EOC tissues compared with normal ovary tissues. We delineated a novel HOXD-AS1/miR-186-5p/ PIK3R3 molecular pathway that regulates cell migration, invasion, and EMT process in EOC. Suppressing HOXD-AS1 can be a possible strategy to improve the clinical outcome of EOC patients.

\section{Additional files}

Additional file 1: Table S1. The sequences of primers used for RT-qPCR assays. (DOCX $12 \mathrm{~kb}$ )

Additional file 2: Figure S1. miR-186-5P inhibition promotes EOC cell migration, invasion and EMT. (PDF $9600 \mathrm{~kb}$ )

Additional file 3: Figure S2. The statistical graph of western-blot. (PDF 4240 kb)

\section{Abbreviations}

3'UTR: 3'Untranslated Region; ARHGAP11A: Rho GTPase activating protein 11A; CA125: Carbohydrate antigen 125; ceRNA: Competing endogenous RNA; Cl: Confidence interval; DAVID: The Database for Annotation, Visualization and Integrated Discovery; E2F8: E2F transcription factor 8; EMT: Epithelial-esenchymal transition; EOC: Epithelial ovarian cancer; FBS: Fetal bovine serum; FDR: False discovery rate; FFPE: Formalin-fixed paraffin-embedded; FIGO: International Federation of Gynecology and Obstetrics; FZD4: Frizzled 4; GAPDH: Glyceraldehyde-3-phosphate dehydrogenase; GEO: Gene Expression Omnibus; GO: Gene Ontology; HOXDAS1: HOXD antisense growth-associated long non-coding RNA; HR: Hazard ratio; KEGG: Kyoto Encyclopedia of Genes and Genomes; IncRNA: Long noncoding RNAs; miRNA: microRNA; PANDAR: The promoter of CDKN1A antisense DNA damage activated RNA; PBS: Phosphate buffer saline; PIK3R3: A regulatory subunit of phosphatidylinositol 3-kinase (PI3K); PTAR: Pro-transition associated RNA; PVT1: Plasmacytoma variant translocation 1; Rac1: Rac family small GTPase 1; RhoC: Ras homolog gene family member C; RT-qPCR: Reverse transcription quantitative PCR;

SAM: Significance analysis of microarray; SD: Standard error; SDS-

PAGE: Sodium dodecyl sulfate- polyacrylamide gel electrophoresis; SFRS2: Arginine/serine-rich 2; SOX4: A member of the SOX (SRY-related HMGbox) gene family; Twist: Twist family bHLH transcription factor 1 ; XIST: Xinactive specific transcript; ZEB1: Zinc finger E-box binding homeobox 1

\section{Acknowledgements}

We would like to thank our colleagues especially Dr. Junjun Li from the department of pathology for their assistance in this project; and thank the staff of Hunan Cancer Hospital / The Affiliated Cancer Hospital of Xiangya School of Medicine; Central South University for their assistance to the study.

\section{Funding}

This study was supported by the National Natural Science Foundation (No. 81201730, No. 81703005 and No.81602480), Key Research and Development Project of Hunan Science and Technology Department (No. 2018SK2126), Key Project of Changsha Science and Technology Bureau (No. kq1706046), Research Project of National Cancer Center Cancer (No.NCC2017A21), Research Project of Health and Family Planning Commission of Hunan Province (No. B2017098), the Natural Science Foundation of Hunan Province (No. 12JJ5073, No. 2017JJ3195, and No. 2018JJ3311), CBRG-NIG grant (NMRC/ BNIG/2037/2015) and OF-YIRG (NMRC/OFYIRG/0076/2018) from National Medical Research Council of Singapore and SingHealth ONCO-ACP grant to YW (NCCRF-OACPCCS-YR2015-Aug-1).

\section{Availability of data and materials}

The IncRNA+mRNA arrays and miRNA arrays data have been deposited into GEO database under the accession number GSE119056. The reviewer's link is provided below. (https://www.ncbi.nlm.nih.gov/geo/query/acc.cgi?acc=GSE119056). An additional set of miRNA expression microarray data, GSE53829, was retrieved from GEO database. 


\section{Authors' contributions}

SSD, RRW, XWS and SL contributed in data acquisition and analysis. JW, KQZ and TH contributed in the collection of patient samples and associated clinical information and analysis. QD and YL contributed in bioinformatics analysis and interpretation of the data and statistical analysis. YZ, YW, HW, XZ and HYR contributed in study design, analysis and interpretation of data, obtaining funding, study supervision and writing the manuscript. All authors read and approved the final manuscript.

\section{Ethics approval and consent to participate}

The research was approved by the Ethics Committee of Hunan Cancer Hospital / The Affiliated Cancer Hospital of Xiangya School of Medicine; Central South University.

\section{Consent for publication}

NA

\section{Competing interests}

The authors declare that they have no competing interests.

\section{Publisher's Note}

Springer Nature remains neutral with regard to jurisdictional claims in published maps and institutional affiliations.

\section{Author details}

${ }^{1}$ Translational Medicine Center, The Affiliated Cancer Hospital of Xiangya School of Medicine, Central South University/Hunan Cancer Hospital, Changsha, China. ${ }^{2}$ Engineering Technology Research Center for diagnosis-treatment and application of tumor liquid biopsy, Changsha, China ${ }^{3}$ Key Laboratory of Radiation Oncology, Department of Radiation Oncology, Hunan Cancer Hospital and The Affiliated Cancer Hospital of Xiangya School of Medicine, Central South University, Changsha, Hunan, China. ${ }^{4}$ The fifth department of gynecological oncology The Affiliated Cancer Hospital of Xiangya School of Medicine, Central South University/Hunan Cancer Hospital, Changsha, China. ${ }^{5}$ Department of Biochemistry and Molecular Biology, Second Military Medical University, Changsha, China. ${ }^{6}$ Division of Cellular and Molecular Research, National Cancer Centre Singapore, Singapore, Singapore.

\section{Received: 24 October 2018 Accepted: 11 February 2019}

\section{Published online: 01 March 2019}

\section{References}

1. Torre LA, Bray F, Siegel RL, Ferlay J, Lortet-Tieulent J, Jemal A. Global cancer statistics, 2012. CA Cancer J Clin. 2015;65(2):87-108

2. Fujiwara K, McAlpine JN, Lheureux S, Matsumura N, Oza AM. Paradigm shift in the management strategy for epithelial ovarian Cancer. Am Soc Clin Oncol Educ Book. 2016;35:e247-57.

3. Nasioudis D, Sisti G, Kanninen TT, Holcomb K, Di Tommaso M, Fambrini M, Witkin SS. Epidemiology and outcomes of squamous ovarian carcinoma; a population-based study. Gynecol Oncol. 2016;141(1):128-33.

4. Siegel RL, Miller KD, Jemal A. Cancer statistics, 2016. CA Cancer J Clin. 2016; 66(1):7-30.

5. Kalluri R, Weinberg RA. The basics of epithelial-mesenchymal transition. J Clin Invest. 2009;119(6):1420-8.

6. Nieto MA, Huang RY, Jackson RA, Thiery JP. Emt: 2016. Cell. 2016;166(1):21-45.

7. Polyak K, Weinberg RA. Transitions between epithelial and mesenchymal states: acquisition of malignant and stem cell traits. Nat Rev Cancer. 2009;9(4):265-73.

8. Acloque H, Adams MS, Fishwick K, Bronner-Fraser M, Nieto MA. Epithelialmesenchymal transitions: the importance of changing cell state in development and disease. J Clin Invest. 2009;119(6):1438-49.

9. Zheng X, Carstens JL, Kim J, Scheible M, Kaye J, Sugimoto H, Wu CC, LeBleu VS, Kalluri R. Epithelial-to-mesenchymal transition is dispensable for metastasis but induces chemoresistance in pancreatic cancer. Nature. 2015; 527(7579):525-30

10. Fischer KR, Durrans A, Lee S, Sheng J, Li F, Wong ST, Choi H, El Rayes T, Ryu S, Troeger J, et al. Epithelial-to-mesenchymal transition is not required for lung metastasis but contributes to chemoresistance. Nature. 2015;527(7579):472-6.

11. Liang SQ, Marti TM, Dorn P, Froment L, Hall SR, Berezowska S, Kocher G, Schmid RA, Peng RW. Blocking the epithelial-to-mesenchymal transition pathway abrogates resistance to anti-folate chemotherapy in lung cancer. Cell Death Dis. 2015;6:e1824.
12. Huang $L, W u R L, X u A M$. Epithelial-mesenchymal transition in gastric cancer. Am J Transl Res. 2015:7(11):2141-58.

13. Zhang PF, Li KS, Shen YH, Gao PT, Dong ZR, Cai JB, Zhang C, Huang XY, Tian MX, Hu ZQ, et al. Galectin-1 induces hepatocellular carcinoma EMT and sorafenib resistance by activating FAK/PI3K/AKT signaling. Cell Death Dis. 2016;7:e2201.

14. Liu S, Sun J, Cai B, Xi X, Yang L, Zhang Z, Feng Y, Sun Y. NANOG regulates epithelial-mesenchymal transition and chemoresistance through activation of the STAT3 pathway in epithelial ovarian cancer. Tumour Biol. 2016;37(7):9671-80.

15. Huarte M. The emerging role of IncRNAs in cancer. Nat Med. 2015;21(11):1253-61.

16. Wang Y, Lee CG. MicroRNA and cancer--focus on apoptosis. J Cell Mol Med. 2009;13(1):12-23.

17. Chen DL, Chen LZ, Lu YX, Zhang DS, Zeng ZL, Pan ZZ, Huang P, Wang FH, $\mathrm{Li} Y \mathrm{H}$, Ju HQ, et al. Long noncoding RNA XIST expedites metastasis and modulates epithelial-mesenchymal transition in colorectal cancer. Cell Death Dis. 2017:8(8):e3011.

18. Chang Z, Cui J, Song Y. Long noncoding RNA PVT1 promotes EMT via mediating microRNA-186 targeting of Twist1 in prostate cancer. Gene. 2018;654:36-42.

19. Barabasi AL, Oltvai ZN. Network biology: understanding the cell's functional organization. Nat Rev Genet. 2004;5(2):101-13.

20. Salmena L, Poliseno L, Tay Y, Kats L, Pandolfi PP. A ceRNA hypothesis: the Rosetta stone of a hidden RNA language? Cell. 2011;146(3):353-8.

21. Liu J, Matulonis UA. New strategies in ovarian cancer: translating the molecular complexity of ovarian cancer into treatment advances. Clin Cancer Res. 2014:20(20):5150-6.

22. Davidson B, Trope CG. Ovarian cancer: diagnostic, biological and prognostic aspects. Womens Health (Lond). 2014;10(5):519-33.

23. Ombrato L, Malanchi I. The EMT universe: space between cancer cell dissemination and metastasis initiation. Crit Rev Oncog. 2014;19(5):349-61.

24. Singh A, Settleman J. EMT, cancer stem cells and drug resistance: an emerging axis of evil in the war on cancer. Oncogene. 2010;29(34):4741-51.

25. Kun ZYX. Chemoresistance to CDDP induces EMT through activation of RAC1 in SKOV3 human ovarian Cancer cells. Prog Biochem Biophys. 2015;42(2):195-6.

26. Wu DD, Chen $X$, Sun KX, Wang LL, Chen S, Zhao Y. Role of the IncRNA ABHD11-AS1 in the tumorigenesis and progression of epithelial ovarian cancer through targeted regulation of RhoC. Mol Cancer. 2017;16(1):138,

27. Liang $H, Y u T$, Han $Y$, Jiang $H$, Wang C, You T, Zhao X, Shan H, Yang R, Yang $L$, et al. LncRNA PTAR promotes EMT and invasion-metastasis in serous ovarian cancer by competitively binding miR-101-3p to regulate ZEB1 expression. Mol Cancer. 2018;17(1):119.

28. Wang $H$, Fang $L$, Jiang J, Kuang $Y$, Wang B, Shang $X$, Han P, Li Y, Liu M, Zhang $Z$, et al. The cisplatin-induced IncRNA PANDAR dictates the chemoresistance of ovarian cancer via regulating SFRS2-mediated p53 phosphorylation. Cell Death Dis. 2018;9(11):1103.

29. Zhong Y, Gao D, He S, Shuai C, Peng S. Dysregulated expression of long noncoding RNAs in ovarian Cancer. Int J Gynecol Cancer. 2016;26(9):1564-70.

30. Lu S, Zhou J, Sun Y, Li N, Miao M, Jiao B, Chen H. The noncoding RNA HOXD-AS1 is a critical regulator of the metastasis and apoptosis phenotype in human hepatocellular carcinoma. Mol Cancer. 2017;16(1):125.

31. Wang H, Huo X, Yang XR, He J, Cheng L, Wang N, Deng X, Jin H, Wang N, Wang $C$, et al. STAT3-mediated upregulation of InCRNA HOXD-AS1 as a ceRNA facilitates liver cancer metastasis by regulating SOX4. Mol Cancer. 2017;16(1):136.

32. Chen Y, Zhao F, Cui D, Jiang R, Chen J, Huang Q, Shi J. HOXD-AS1/miR-130a sponge regulates glioma development by targeting E2F8. Int J Cancer. 2018;142(11):2313-22.

33. Wang $H$, Shen $Q$, Zhang $X$, Yang C, Cui S, Sun Y, Wang L, Fan X, Xu S. The long non-coding RNA XIST controls non-small cell lung Cancer proliferation and invasion by modulating miR-186-5p. Cell Physiol Biochem. 2017:41(6):2221-9.

34. Mei Q, Li X, Zhang K, Wu Z, Li X, Meng Y, Guo M, Luo G, Fu X, Han W. Genetic and methylation-induced loss of miR-181a2/181b2 within chr9q33.3 facilitates tumor growth of cervical Cancer through the PIK3R3/Akt/FoxO signaling pathway. Clin Cancer Res. 2017:23(2):575-86.

35. Yu T, Li J, Yan M, Liu L, Lin H, Zhao F, Sun L, Zhang Y, Cui Y, Zhang F, et al. MicroRNA-193a-3p and -5p suppress the metastasis of human non-smallcell lung cancer by downregulating the ERBB4/PIK3R3/mTOR/S6K2 signaling pathway. Oncogene. 2015;34(4):413-23

36. Peng YP, Zhu Y, Yin LD, Wei JS, Liu XC, Zhu XL, Miao Y. PIK3R3 promotes metastasis of pancreatic Cancer via ZEB1 induced epithelial-mesenchymal transition. Cell Physiol Biochem. 2018;46(5):1930-8.

37. Wang $Y$, Zhang W, Wang Y, Wang S. HOXD-AS1 promotes cell proliferation, migration and invasion through miR-608/FZD4 axis in ovarian cancer. Am J Cancer Res. 2018;8(1):170-82. 